\title{
Relire Perec, Actes du Colloque de Cerisy, sous la direction de Christelle REGGIANI
}

\section{Laura Brignoli}

\section{OpenEdition}

\section{Journals}

\section{Édition électronique}

URL : https://journals.openedition.org/studifrancesi/12111

DOI : 10.4000/studifrancesi. 12111

ISSN : 2427-5856

\section{Éditeur}

Rosenberg \& Sellier

\section{Édition imprimée}

Date de publication : 1 avril 2018

Pagination : 166-167

ISSN : 0039-2944

\section{Référence électronique}

Laura Brignoli, «Relire Perec, Actes du Colloque de Cerisy, sous la direction de Christelle REGGIANI 》, Studi Francesi [En ligne], 184 (LXII | I) | 2018, mis en ligne le 03 juillet 2018, consulté le 17 novembre 2021.

URL : http://journals.openedition.org/studifrancesi/12111; DOI : https://doi.org/10.4000/ studifrancesi. 12111

Ce document a été généré automatiquement le 17 novembre 2021.

\section{(c) (i) (9)}

Studi Francesi è distribuita con Licenza Creative Commons Attribuzione - Non commerciale - Non opere derivate 4.0 Internazionale. 


\title{
Relire Perec, Actes du Colloque de Cerisy, sous la direction de Christelle REGGIANI
}

\author{
Laura Brignoli
}

\section{RÉFÉRENCE}

Relire Perec, Actes du Colloque de Cerisy, sous la direction de Christelle REGGIANI, Rennes, Presses Universitaires de Rennes, 2016, «La Licorne» 122, 426 pp.

«Je n'écris que pour être relu»: la phrase célèbre de Gide, qui console bon nombre d'écrivains s'estimant mal lus par leurs contemporains, n'aurait peut-être jamais eu l'adhésion de Perec, qui se souciait moins de sa postérité que de la jubilation présente de l'acte d'écriture. Mais la richesse même de l'œuvre de cet auteur, désormais grand classique, comme l'affirme dans la présentation Christelle REGGIANI, ne cesse de susciter de nouvelles approches critiques: les actes du Colloque de Cerisy qui a eu lieu du 13 au 20 juillet 2015 le montrent bien. Ce volume de relectures perspicaces s'engage à ouvrir des perspectives nouvelles dans l'œuvre perecquienne, comme le fait Claude BURGELIN (Le silence de Perec, pp. 15-26) en cherchant les traces d'un Perec plus profond que l'écrivain, enfoui derrière non seulement le manque ou les chiffres, mais au niveau structurel dans la cassure, et au niveau thématique dans le faux. L'élaboration des projets qui sont souvent à la base des œuvres perecquiennes crée un dialogue entre les deux instances auctoriales - «le sujet et l'opérateur» - auxquelles il faut ajouter le lecteur (Adrien CHASSAIN, Perec et la rhétorique du projet, pp. 27-39). Raoul DELEMAZURE (Le geste intertextuel de Georges Perec, pp. 41-57) analyse l'évolution de la pratique intertextuelle à partir de la légitimation de sa pratique scripturale jusqu'au traitement de la «névrose du vide». Allison JAMEs (Perec aux marges de la fiction, pp. 59-72) s'interroge sur le rapport entre le réel et la fiction et laisse émerger «la complexité de l'aspect documentaire de l'œuvre de Perec». Maryline HECK, fidèle à sa quête des 
éléments apparemment inexistants chez Perec (rappelons son livre Perec, le corps à la lettre, 2012), trouve dans l'infra-ordinaire la dimension morale et politique qui semblait faire défaut (Pour un Perec politique, pp. 73-88). Peter CONSENSTEIN (L'identité juive de Georges Perec, pp. 89-104) parcourt les étapes de la prise de conscience de sa judéité de la part de Perec, et de son expression poétique. La longue étude de Jean-Jacques THOMAS (Perec en Amérique, pp. 105-139) rend compte d'une enquête concernant les différents aspects de la présence de Perec sur le sol américain.

2 Les "Questions de poétique», abordées dans la deuxième partie, s'ouvrent avec une analyse des œuvres de Perec classées en trois ensembles distincts sur la base des types de chapitres dans lesquels il a choisi de les découper (Nathalie DANCY, Esthétique du chapitre dans l'œeuvre de Georges Perec, pp. 143-160). Les noms des lieux (Derek SCHILLING, "Entre sens et nevers»: géopoétique du nom de lieu, pp. 161-181) et des personnages (Véronique MONTÉMONT, Onomastique perecquienne, pp. 183-199) participent les premiers à l'ambition «encyclopédique» des fictions perecquiennes, les autres au sentiment d'incertitude des origines et à l'exigence contradictoire de définir et d'effacer, tout en restant «l'un des leviers de cohérence les plus subtils». Steen Bille JøRGENSEN (Lire "La Belle et la Bête": des jeux d'échelles, des modèles narratifs et des formules littérales dans l'œuvre de Perec, pp. 201-216) clôt la section en analysant dans le chapitre LXXIII de La vie mode d'emploi la reprise du modèle narratif du conte de madame Leprince de Beaumont.

Les «(Re)lectures» de la troisième partie abordent les œuvres les plus connues de Perec, comme La disparition, dont Hermes SALCEDA (Contrainte et mémoire dans "La disparition", pp. 219-231) propose une nouvelle interprétation en analysant les mécanismes constitutifs de ce texte, et Yû MAEYAMA (Les notes préparatoires à "La disparition" de Georges Perec: genèse de la saga d'une famille brisée, pp. 233-258) une étude génétique, tout comme Danielle constantin à propos de La vie mode d'emploi (Les manuscrit de "La vie mode d'emploi": des pistes à explorer, pp. 326-346), tandis que Maxime DECOUT ("Je rêve de mes terres d'Ellesmère», pp. 259-270) dévoile les implications autobiographiques d'une des contraintes les plus dures utilisées par Perec, le monovocalisme des Revenentes. L'intérêt pour les explorations autobiographiques caractérise les études de Marie BONNOT, qui analyse la convergence entre contrainte et autobiographie dans La boutique obscure (Une tentative d'épuisement du récit de rêve?, pp. 271-283), et de Dominique MONCOND'HUI, qui prend en examen le parcours qui a conduit à la version définitive d'Espèces d'espaces (Du vide à l'écrit, autoportrait de l'écrivain, pp. 285-305). Jean-Luc JoLY (Petits modes d'emploi: une communication-feuilleton, pp. 307-326) met en lumière et propose une interprétation pour des contraintes encore obscures de $L a$ vie mode d'emploi. Christophe REIG (Le(s) Voyage(s) d'hiver(s): fiction centrifuge/fictions transfuges, pp. 347-366) montre, dans la prolifération transfictionnelle du Voyage d'hiver, la reprise du «travail de sape mise en place par Perec lui-même» contre «l'autorité auctoriale» dans un «rituel ambigu de la canonisation/décanonisation de Perec». Enfin Annelies schulte NORDHOLT (Le travail de la mémoire dans "Lieux", pp. 367-379) explore le pouvoir mnémonique des loci et sa fonction pour Perec.

4 La dernière section, reprenant le titre de la première, montre les possibles «Ouvertures» de l'œuvre de Perec. L'étude de Philippe WAHL, intitulée Calet/Perec: essais autographiques (pp. 383-402), aborde la question de l'écriture comme légitimation identitaire, tandis que celle d'Eléonore HAMAIDE-JAGER (Faire $d u n(o) e u f$ avec du vieux: "La disparition" de Perec et "Pool!" de Pascale Petit et Renaud Perrin, pp. 403-416) concerne une 
réécriture de $L a$ disparition destinée aux enfants, dans la prolongation de la perspective ludique qui est une des assises de tout l'œuvre perecquien. 